Annals of Pure and Applied Mathematics

Vol. 16, No. 2, 2018, 287-293

ISSN: 2279-087X (P), 2279-0888(online)

Published on 15 February 2018

www.researchmathsci.org

DOI: http://dx.doi.org/10.22457/apam.v16n2a4

Annals of

Pure and Applied

Mathematics

\title{
Computation of Some Degree Based Topological Indices of Silicates $\left(\mathrm{SiO}_{2}\right)$ Layer
}

\author{
A.Bharali ${ }^{1}$ and Rajdeep Bora ${ }^{2}$ \\ Department of Mathematics \\ Dibrugarh University, Dibrugarh-786004 \\ Email: ${ }^{1}$ a.bharali@dibru.ac.in \\ ${ }^{2}$ Corresponding author. Email: rajdeepbora23@gmail.com
}

Received 1 February 2018; accepted 14 February 2018

\begin{abstract}
Topological Indices is a graph invariant, which is defined from a set of finite graphs to real numbers. A large number of topological indices have been conceived, depending on vertex degrees, which are called degree based topological indices. In this paper it is tried to discuss about some of these degree based topological indices viz., Augmented Zagreb indices, Harmonic index, and the three $\mathrm{S}$ indices in connection with $\mathrm{SiO}_{2}$ layer structurein a graph theoretic prospective.
\end{abstract}

Keywords: Topological index, $\mathrm{SiO}_{2}$ layer structure, Augmented-Zagreb index, Harmonic index, $\mathrm{S}$ indices.

\section{AMS Mathematics Subject Classification (2010): 05 C62}

\section{Introduction}

Topological indices are numerical invariants, associated with the topological characterization of a compound. By topological characterization, we mean that these indices relate to certain properties of the compound including the toxicological, physicochemical, pharmacological etc. properties. From chemistry's point of view, compounds' properties give a measure of how a certain compound will behave and react but the process is both time, energy consuming and sometimes not possible due to financial constraints. On the other hand Chemical graph theory provides an easy alternative to this limitation. Mathematically a topological index is a function from a set $G$ of finite graphsto real numbers i.e., Top : $G \rightarrow$ R. Calculating topological indices is not only useful for verifying the existing properties of chemical compound but also for calculating new applications of naturally occurring molecules and more interestingly these can be used to design new compounds with specifically required properties [1].

Chemical graph theory has an important role in the development of the chemical sciences by using topological indices. The first distance based topological index was proposed by Wiener in 1947 for modeling physical properties of alkanes, and till date, many topological indices were defined by chemists and mathematicians and so many properties of chemical structures were studied with these indices. One of such recent 
A.Bharali and Rajdeep Bora

index is S-index, which was proposed in 2017, by Ediz [3]. In 2016, Farrukhet. al., have computed different versions of Randić index [12], Sum-connectivity index [14] of $\mathrm{SiO}_{2}$ layer structure [5]. They have also obtained [6] various results for $\mathrm{SiO}_{2}$ layer structure in connection with $\mathrm{ABC}$ index [4] and Geometric-Arithmetic index [7]. In [9][10], Kulli discussed new multiplicative version of connectivity indices in nanotubes. In 2017, Kulli also proposed new arithmetic-geometric indices based on different vertex parameters instead of degree [11].

The rest of the paper is organized as follows: in next section we discuss few very popular topological indices and also present the definition of $S$ index. In section 3, we discuss the $\mathrm{SiO}_{2}$ and its layer structure as a graph structure.Then in section 4 and section 5 , we present some new results which are obtained during our study.

\section{Some vertex degree based topological indices}

In the literature of chemical graph theory, topological indices are used to study molecule by studying the correlation between some property of the molecule and the values of certain index. These indices are based on degree of vertices of a graph. Some of such popular degree-based topological indices are discussed in this section.

\subsection{Augmented-Zagreb index}

The augmented Zagreb index of a graph $\mathrm{G}$ is defined to be

$$
A Z I(G)=\sum_{u v \in E(G)}\left(\frac{d_{u}(G) d_{v}(G)}{d_{u}(G)+d_{v}(G)-2}\right)^{3} .
$$

This index is demonstrated to be an important molecular descriptor [8], which draws limited attention from the researchers.

\subsection{Harmonic Index}

In 1980s, Siemion Fajtlowicz for the first time introduced the vertex-degree-based index

$$
\mathrm{HI}(\mathrm{G})=\sum_{\mathrm{uv} \in \mathrm{E}(\mathrm{G})} \frac{2}{\mathrm{~d}_{\mathrm{u}+\mathrm{d}_{\mathrm{v}}}} .
$$

In 2012 Zhong reintroduced this index and called it Harmonic Index [13].

\section{3. $\mathrm{S}$ indices}

The set of all vertices which adjacent to vertex $\mathrm{v}$ is called the open neighborhood of $\mathrm{v}$ and is denoted by $N(v)$. If we add the vertex $\mathrm{v}$ to $N(v)$ then we get the closed neighborhood of $\mathrm{v}, \mathrm{N}[\mathrm{v}]$. For a vertex v, $S_{v}=\sum_{u \in N(v)} \operatorname{deg}(u)$ is the sum degree of v or briefly "sum degree" and $M_{v}=\prod_{u \in N(v)} \operatorname{deg}(u)$ is the multiplication degree of $\mathrm{v}$ or briefly "multiplication degree".

Definition 2.8.1. [3] The $\mathrm{S}$ degree of a vertex $v$ of a simple connected graph $\mathrm{G}$ is defined as

$$
S(v)=\left|M_{v}-S_{v}\right|
$$

Using this $S$ degree of a vertex, three indices similar to Zagreb indices [2] are proposed by Ediz [3], which are as follows.

Definition 2.8.2. [3] The first $\mathrm{S}$ index of a simple connected graph $\mathrm{G}$ is defined as 
Computation of Some Degree Based Topological Indices of Silicates $\left(\mathrm{SiO}_{2}\right)$ Layer

$$
S^{1}(G)=\sum_{v \in V(G)} \mathrm{S}(\mathrm{v})^{2} .
$$

Definition 2.8.3. [3] The second $\mathrm{S}$ index of a simple connected graph $\mathrm{G}$ is defined as

$$
S^{2}(G)=\sum_{u v \in E(G)} S(u) S(v) \text {. }
$$

Definition 2.8.4. [3] The third $\mathrm{S}$ index of a simple connected graph $\mathrm{G}$ is defined as

$$
S^{3}(G)=\sum_{u v \in E(G)}[S(u)+S(v)] .
$$

\section{Layer structure of $\mathrm{SiO}_{2}$}

We know that the silicates $\left(\mathrm{SiO}_{2}\right)$ consist of one silicon ion and four oxygen ions, as shown in Figure 1.

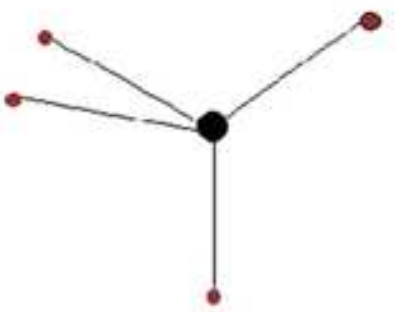

Figure 1: $\mathrm{SiO}_{2}$ ion

Silicates form different networks, based on their polymerization but the networks are usually motivated by the original silicates network. The $\mathrm{SiO}_{2}$ layer structure, on the other hand is the original silicates structure. Silicates, in its naturally occurring form, forms an octagon of sorts, as shown in Figure 2.

And finally, these octagons join together to form the $\mathrm{SiO}_{2}$ layer structure. We define the rows as the number of lines of vertical octagons and columns as the number of lines of horizontal octagons. We denote the number of rows with $p$ and the number of columns with $q$. For better understanding, Figure 3 gives the $\mathrm{SiO}_{2}$ layer structure with $p=$ 4 and $q=5$. The concept can be extended to any number of rows and columns.

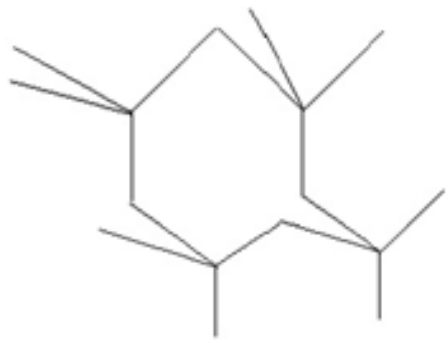

Figure 2: $\mathrm{SiO}_{2}$ octagon 


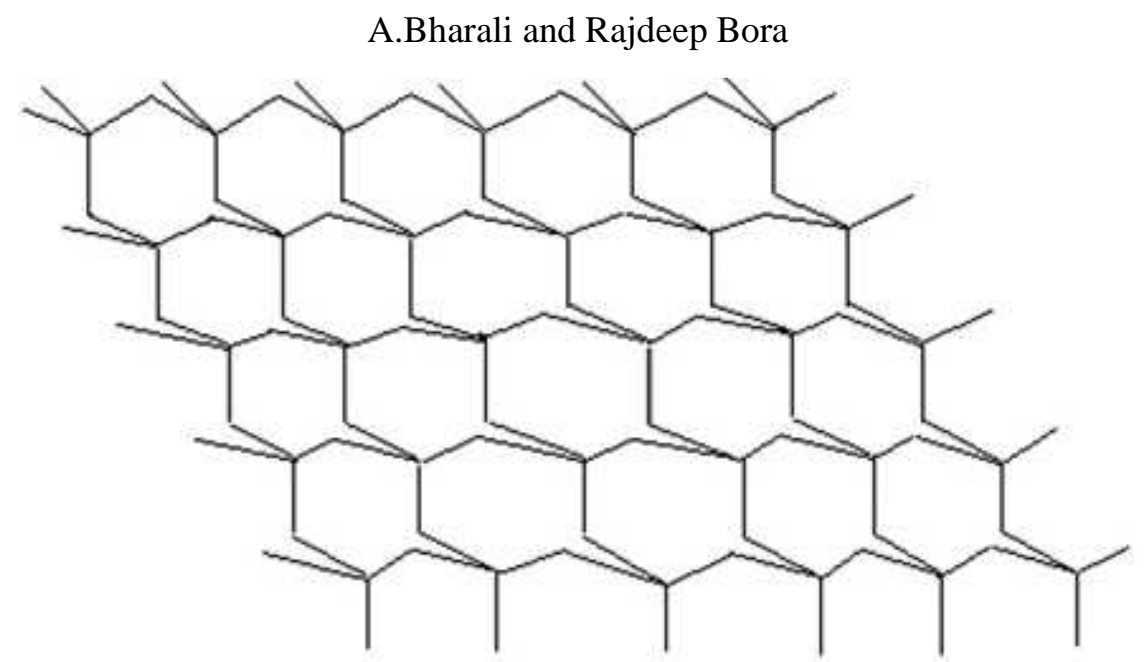

\section{Results and discussion}

Figure 3: $\mathrm{SiO}_{2}(4,5)$

\subsection{Computation of $\mathrm{AZI}, \mathrm{HI}$ of $\mathrm{SiO}_{2}(\mathrm{p}, \mathrm{q})$ - layer structure}

The values of topological indices such as AZI $\left(\mathrm{SiO}_{2}\right), \mathrm{GA}\left(\mathrm{SiO}_{2}\right)$ and $\mathrm{ABC}\left(\mathrm{SiO}_{2}\right)$ are calculated for the (p, q)- layer structure of $\mathrm{SiO}_{2}$ for arbitrary values of $\mathrm{p}$ and $\mathrm{q}$. We have seen in Figure 3. There are two types of edges in the structure of $\mathrm{SiO}_{2},(1,4)$ type i.e., end vertices of the edge are of degree 1 and 4 and $(2,4)$ type i.e., end vertices of the edge are of degree 2 and 4 . The detail of it is summarized in Table 4.1.

\section{Table 4.1:}

$\begin{array}{cc}\text { Type of Edges } & \text { Number of Edges } \\ (1,4) & 2 p+2 q+4 \\ (2,4) & 2 p+2 q+4 p q\end{array}$

Theorem 4.1.1. The augmented $\mathrm{Zagreb}$ index of $\mathrm{SiO}_{2}(\mathrm{p}, \mathrm{q})$ layer structure is given by

Proof: $A Z I(G)=\sum_{u v \in E(G)}\left(\frac{d_{u}(G) d_{v}(G)}{d_{u}(G)+d_{v}(G)-2}\right)^{3}$

$$
\mathrm{AZI}\left(\mathrm{SiO}_{2}\right)=560 \frac{(\mathrm{p}+q)}{27}+32 p q+\frac{256}{27}
$$

Now replacing the values from the table 4.1, we have

$$
\begin{aligned}
\operatorname{AZI}\left(\mathrm{SiO}_{2}\right)=\sum_{u v \in(1,4)}\left(\frac{1 \times 4}{1+4-2}\right)^{3}+\sum_{u v \in(2,4)}\left(\frac{2 \times 4}{2+4-2}\right)^{3} \\
=(2 p+2 q+4) \times\left(\frac{4}{3}\right)^{3}+(2 p+2 q+4 p q) \times 8 \\
=(2 p+2 q+4) \times\left(\frac{64}{27}+8\right)+32 p q+\frac{256}{27} \\
=560 \frac{(\mathrm{p}+q)}{27}+32 p q+\frac{256}{27} .
\end{aligned}
$$

Theorem 4.1.2. The Harmonic index of $\mathrm{SiO}_{2}(\mathrm{p}, \mathrm{q})$ layer structure is given by

$$
\mathrm{HI}\left(\mathrm{SiO}_{2}\right)=22 \frac{(\mathrm{p}+q)}{15}+\frac{4}{3} p q+\frac{8}{5} .
$$

Proof: $H I(G)=\sum_{u v \in E(G)} \frac{2}{d_{u+} d_{v}}$, now replacing the values from the table 4.1, we have 
Computation of Some Degree Based Topological Indices of Silicates $\left(\mathrm{SiO}_{2}\right)$ Layer

$$
\begin{aligned}
\mathrm{HI}\left(\mathrm{SiO}_{2}\right) & =\sum_{u v \in(1,4)}\left(\frac{2}{1+4}\right)+\sum_{u v \in(2,4)}\left(\frac{2}{2+4}\right) \\
& =(2 p+2 q+4) \times\left(\frac{2}{5}\right)+(2 p+2 q+4 p q) \times\left(\frac{2}{6}\right) \\
& =22 \frac{(\mathrm{p}+q)}{15}+\frac{4}{3} p q+\frac{8}{5} .
\end{aligned}
$$

\subsection{Computation of the $\mathrm{S}$ indices of $\mathrm{SiO}_{2}(p, q)$ layer structure}

From the knowledge of the edges present in the structure, we can easily classify the set of vertices in three main categories based on their degrees viz., degree 1, degree 2 and degree 4 . Then based on the neighborhood of a vertex we can further classify these vertices into five categories. In case of vertices of degree 1 and 2, from Figure 3, we can observe that a degree 1 vertex is adjacent to a degree 4 vertex and a degree 2 vertex is adjacent to two degree 4 vertices. We call them Type I and Type II respectively. Again vertex of degree 4 has three types of adjacent vertices, so we call them Type III, Type IV, and Type V. A Type III vertex is adjacent to two degree 1 vertices and two degree 2 vertices, a Type IV vertex is adjacent to one degree 1 vertex and three degree 2 vertices, and a Type $\mathrm{V}$ vertex is adjacent to four degree 2 vertices. An account of all the types vertices present in the structure is given in Table 4.2.

Degree of vertex

1

2

4

Type

I
II
III

IV

V
Table 4.2:

S degree

Number of such vertices

$$
\begin{array}{cc}
0 & 2(p+q+2) \\
8 & p+q+2 p q \\
2 & 4 \\
1 & 2(p+q-2) \\
8 & (p-1)(q-1)
\end{array}
$$

Theorem 4.2.1. The first $\mathrm{S}$ index of $\mathrm{SiO}_{2}(\mathrm{p}, \mathrm{q})$ layer structure is given by

$$
S^{1}\left(\mathrm{SiO}_{2}\right)=2(p+q)+192 p q+76
$$

Proof: We know that the first $\mathrm{S}$ index of any connected graph $\mathrm{G}$ is

$$
S^{1}(G)=\sum_{v \in V(G)} \mathrm{S}(\mathrm{v})^{2}
$$

Now,

$$
\begin{aligned}
& S^{1}\left(\mathrm{SiO}_{2}\right)=\sum_{v \in \text { TypeI }} S(v)^{2}+\sum_{v \in \text { TypeII }} S(v)^{2}+\sum_{v \in \text { TypeIII }} S(v)^{2}+\sum_{v \in \text { TypeIV }} S(v)^{2} \\
& +\sum_{v \in \text { TypeV }} S(v)^{2} \\
& =0^{2} \times 2(p+q+2)+8^{2}(p+q+2 p q)+2^{2} \times 4+ \\
& 1^{2} \times 2(p+q-2)+8^{2}(p-1)(q-1) \\
& =8^{2}(p+q+2 p q)+16+2(p+q-2)+8^{2}(p q-p-q+1)
\end{aligned}
$$


A.Bharali and Rajdeep Bora

$$
\begin{aligned}
& =2(p+q)+64 \times 3 p q+16-4+64 \\
& =2(p+q)+192 p q+76
\end{aligned}
$$

Theorem 4.2.2. The second $\mathrm{S}$ index of $\mathrm{SiO}_{2}(\mathrm{p}, \mathrm{q})$ layer structure is given by

$$
S^{2}\left(\mathrm{SiO}_{2}\right)=256 p q-208(p+q)+288
$$

Proof: We know that the second $\mathrm{S}$ index of any connected graph $\mathrm{G}$ is

$$
S^{2}(G)=\sum_{u v \in E(G)} S(u) S(v)
$$

Then, $S^{2}\left(\mathrm{SiO}_{2}\right)=\sum_{u v \in(1,4)} S(u) S(v)+\sum_{u v \in(2,4)} S(u) S(v)$

$$
=0+\sum_{u v \in(2,4)} S(u) S(v) \text {, }
$$

(Since $S(1)=0$, so $\sum_{u v \in(1,4)} S(u) S(v)=0$.)

$=\sum_{u v \in(2,4)} S(u) S(v)$

Now we can observe that there will be three types of $(2,4)$ edges, which are associated with Type III, Type IV, and Type $V$ vertex of degree 4. In Type III, there are two $(2,4)$ edges associated with each vertex of degree 4 along with two vertices of degree 1. Again in Type IV, there are three $(2,4)$ edges associated with each vertex of degree 4 along with one vertex of degree 1, and in Type $V$, we have four edges associated with the vertex of degree 4. So,

$$
\begin{aligned}
& S^{2}\left(\mathrm{SiO}_{2}\right)=\sum_{(2,4) \in \text { TypeIII }}(8 \times 2)+\sum_{(2,4) \in \text { TypeIV }}(8 \times 1)+\sum_{(2,4) \in \text { TypeV }}(8 \times 8) \\
& =(2 \times 4)(8 \times 2)+3 \times 2(p+q-2)(8 \times 1)+ \\
& 4 \times(p-1)(q-1)(8 \times 8) \\
& =128+48(p+q-2)+256(p q-p-q+1) \\
& =256 p q-208(p+q)+288 \text {. }
\end{aligned}
$$

Theorem 4.2.3. The third $\mathrm{S}$ index of $\mathrm{SiO}_{2}(\mathrm{p}, \mathrm{q})$ layer structure is given by

$$
S^{2}\left(\mathrm{SiO}_{2}\right)=64 p q-8(p+q)+48
$$

Proof: We know that the third $\mathrm{S}$ index of any connected graph $\mathrm{G}$ is

Now,

$$
S^{3}(G)=\sum_{u v \in E(G)}[S(u)+S(v)] .
$$

$$
\begin{aligned}
S^{3}\left(\mathrm{SiO}_{2}\right)= & \sum_{u v \in(1,4)}[S(\mathrm{u})+S(v)]+\sum_{u v \in(2,4)}[S(\mathrm{u})+S(\mathrm{v})] \\
= & \sum_{(1,4) \in T y p e ~ I I I}[S(\mathrm{u})+S(v)]+\sum_{(1,4) \in T y p e I V}[S(\mathrm{u})+S(v)]+\sum_{(2,4) \in T y p e I I I}[S(\mathrm{u}) \\
& +S(\mathrm{v})]+\sum_{(2,4) \in \text { Type IV }}[S(\mathrm{u})+S(\mathrm{v})]+\sum_{(2,4) \in \text { Type } V}[S(\mathrm{u})+S(\mathrm{v})]
\end{aligned}
$$

Here, we have again chosen the types of edges based on different types of vertex 4 , because vertex 1 has only one type, i.e., Type $I$ and so on.

$$
\begin{aligned}
= & (2 \times 4)[0+2]+2(p+q-2)[0+1]+(2 \times 4)[8+2]+ \\
& 3 \times 2(p+q-2)[8+1]+4(p-1)(q-1)[8+8] \\
= & 16+2(p+q-2)+80+54(p+q-2)+64(p q-p-q+1) \\
= & 64 p q-8(p+q)+48
\end{aligned}
$$


Computation of Some Degree Based Topological Indices of Silicates $\left(\mathrm{SiO}_{2}\right)$ Layer

\section{Conclusion}

In this study, we have calculated Augmented Zagreb Index, Harmonic Index and three S indices of $\mathrm{SiO}_{2}(p, q)$-layer structure for all values of $p$ and $q$. From the results, we can observe that the expressions are symmetric and hence thechange in the values of $p$ and $q$, will have similar effect on these indices of $\mathrm{SiO}_{2}(p, q)$-layer structure.

Acknowledgement. Authors are grateful to all the anonymous reviewers for their valuable suggestions.

\section{REFERENCES}

1. A/Ali, Z.Raza and A.A.Bhatti, On the augmented Zagreb index, Kuwait J. Sc., 43(2) (2016) 48-63.

2. A.T.Balaban, I.Motoc, D.Bonchev and O.Mckeyan, Design of topological indices, Topics Curr. Chem., 114 (1983) 21.

3. S.Ediz, On $\mathrm{S}$ degrees of vertices and $\mathrm{S}$ indices of graph, Science Journal of Analytical Chemistry, 5(6) (2017) 86-89.

4. E.Estrada, An atom-bond connectivity index: modeling the enthalpy of formation of alkanes, Indian J. of Chem., 37A (10) (1998) 849-855.

5. F.Farrukh, S.Hafi, R.Farooq and Mohammad Reza Farahani, Calculating some topological indices of $\mathrm{SiO}_{2}$ layer structure, Journal of Informatics and Mathematical Sciences, 8(3) (2016) 181-187.

6. F.Farrukh, et. al., On atom-bond connectivity and geometric-arithmetic indices of $\mathrm{SiO}_{2}$ layer structure, Moroccan Journal of Chemistry, 5(2) (2017) 384-390.

7. I.Gutman, Degree-based topological indices, Croatica Chemica Acta, 86(4) (2013) 351-361.

8. Y.Huang, Augmented Zagreb index of connected graphs, MATCH Commun. Math.Comput. Chem., 67(2) (2012) 483-494.

9. V.R.Kulli, Multiplicative connectivity indices of certain nanotubes, Annals of Pure and Applied Mathematics, 12(2) (2016) 169-176

10. V.R.Kulli, Two new multiplicative atom bonds connectivity indices, Annals of Pure and Applied Mathematics, 13(1) (2017) 1-7.

11. V.R.Kulli, New arithmetic-geometric indices, Annals of Pure and Applied Mathematics, 13(2) (2017) 165-172.

12. M.Randic, Characterization of molecular branching, J. Am. Chem. Soc., 97 (23) (1975) 6609-6615.

13. L.Zhong, The Harmonic index of graph, Appl. Math. Lett., 25(3) (2012) 561-566.

14. B.Zhou and N.Trinajstic, On a novel connectivity index, J. Math. Chem., 46(4) (2009) 1252-1270. 